\title{
Analysis of Space Constituents of Clothing Dimensional Modeling Design
}

\author{
Lanrong $\mathrm{Yu}$ \\ Weihai Vocational College, Weihai, Shandong, 264200, China
}

\begin{abstract}
With the rapid social and economic development, people's living standards and quality has been significantly improved, the requirements of clothing has also improved for the modern society, the clothing is not only used to cover the body or a means of cold, it is also an important tool to show temperament, highlight the personality, shape the image at the same time. This article mainly analyzes and studies the three-dimensional design of the garment from three aspects: the outline of three-dimensional composition, the particularity of fashion design and the spatial elements of three-dimensional design of clothing, it has an important significance and role on the rationality and comfortableness of three-dimensional modeling design.
\end{abstract}

Keywords: garment dimensional modeling design; space composition; factor analysis

\section{Introduction}

Three-dimensional modeling of clothing design is the use of three-dimensional modeling means, the performance of the organic clothing space and the design of a style of clothing style of a way. For the space of clothing, it is not only the three-dimensional of the garment itself, but also the three-dimensional which is highlighted in the design. It is an important form of the visual modeling art, and reflects a certain space visual effect. 


\section{The overview of three-dimensional composition art}

The law of three-dimensional composition is the process of realizing the transition from two-dimensional plane image to three-dimensional space, and there is a certain difference between them, but at the same time they are interrelated. As an independent study of shape design and form creation, threedimensional composition on the one hand requires the use of painting art and patterns on the plane to create a certain sense of space and image, on the other hand, in the three-dimensional design and three-dimensional art contains all of the plastic arts type [1]. It is characterized by defining and occupying the space by the entity, then forming the new visual product and the environment together with the space, which is the so-called space art.

\section{The Particularity of Fashion Design}

\subsection{The particularity of the object}

Fashion modeling and statues, paintings and other art forms have a certain distinction between the designer can not create an abstract form or the real form of nature to show directly, but also not the same architectural style, in addition to the need to meet people's activities and living Space, but also need to have a certain performance and flexibility, clothing shape is based on the human form in the most natural state, through a variety of clothing materials on the human body shape reconstruction and performance. For the human body, belong to the life of the multi-dimensional activities of the body, different surfaces together constitute its surface, this will to a certain extent, limit and restrict the shape of clothing, the use of its material requirements are relatively high [2]. Another clothing material is mainly flat materials, such as fabric, if you want to design a three-dimensional modeling, you need to complete the plane material and three-dimensional modeling changes. Dimensional modeling design, on the one hand contains the colour, flower-shaped design and other two-dimensional design factors; the other hand, also contains multi-dimensional form, which is mainly after clothing to show a certain shape. The transformation between these two different forms, but also in the three-dimensional modeling of clothing a unique process, but also with other plastic arts appear differences in the main aspects.

\subsection{The Particularity of the material}

As a kind of artistic creation, fashion design is the background of Chinese and Western art history, and then fully combines the art of image perception art, colour matching art and space plastic art. And other forms of design is different, the role of fashion design object is fabric, if only from the perspective of modeling, then, clothing can also be called the fabric of the sculpture. And space art form the same is that the basic framework of clothing or support shape is the human body, is an important support in the clothing. For example, in the 
"Madame Butterfly" show field, models who fully show folding fan, pine, cherry, lanterns and other classic elements, from the fabric is also fully demonstrated the origami art [3].

\section{The space components of clothing three-dimensional modeling design}

\subsection{The space analysis outside the clothing}

Clothing outside the space refers to the clothing itself, the other all the external space, through the outer space of the garment shape, people can get the feeling of beauty, while the human body modification and landscaping. Its characteristics are generally considered to be largely ambiguous. After the three-dimensional design of clothing, often change the shape of clothing, resulting in outer space distortion, which is the so-called outward expansion of power, people from the visual access to dynamic enjoyment [4]. In the design of such clothing is the time, the designer must be able to accurately grasp the psychological characteristics of consumers. For the outer space design, breaking the design practices, but also to some extent reflects the current habit of boredom and resentment of the people, which is the habit of modern people and welcome the distortion of space design reasons. In addition, environmental factors, microscopic factors and macroscopic factors also affect the design of outer space distortion to a certain extent.

No matter what period of clothing, are bound to be severely affected by natural weather and this effect has the certain continuity. If people's clothing and the surrounding environment is quite different, it will inevitably be the exclusion of the environment. In different atmospheres and occasions, will form a different atmosphere and form, thus affecting people's clothing, and if the geographical location also has some differences, the clothing mix will be different, such as domestic and foreign, Villages and cities in different regions, people's clothing with different. So designers in the design of the clothing when the environment will be fully taken into account the climatic conditions. At the same time for the time factor, but also to a certain extent, the clothing decoration techniques, fabric and shape have an impact. In fashion design, people are important to the core, so the designer in the fashion design, will inevitably be summed up in advance and summarized a variety of factors on the impact of clothing, a clear design orientation, so that the design of the clothing before will have the necessary targeted [5].

The concept of human life mainly by the individual's actual needs and lifestyle and attitude composition in the community because of people's minds has a certain degree of difference, so that people's attitude to life will be different, and then their style of dress impact. At the same time because of people's clothing patterns, shape and colour of the degree of love is different, in the choice of clothing when it will inevitably reflect a greater difference, the differences can be through the clothing colour, style and other factors change performance come out. 
If people want to get comfortable, then wearing the clothes needs to adapt to the social environment. Clothing designers need to take full account of the practicality of clothing, on the one hand clothing on the social environment changes have some adaptability, on the other hand clothing on population distribution, geographical weather and other natural factors have some adaptability. In addition, clothing designers also need to fully aware of people's attitudes, lifestyles and so on with the times, seasons and age changes have a certain change. With the social value standards, social values and changes in the level of production technology, people's dressing habits will also change. Socioeconomic development, and changes in the country's political system, will to some extent affect the clothing design, fabric and style. Social unrest and political change will easily lead to changes in clothing, with the degree of economic development and the degree of opening up the political environment will gradually show a variety of clothing styles, and moral norms and social and cultural concepts to a certain extent, the prevalence of clothing.

\subsection{The space analysis within the clothing}

Garment space refers to the clothes and the body, clothing and the formation of a certain distance between the body, the existence of clothing space to ensure the air circulation, so clothing also has a cooling and warm features to improve the wearer comfort, While clothing space will also affect the varying degrees of the wearer's aesthetic needs and activities of attitude. In the process of movement, clothing space will have a corresponding change and it is because the wearer's behaviour involved in the clothing space can reflect its practical function. In addition, by building the shape of space within the clothing characteristics, the basic activities of the wearer's needs can be very good to meet.

In the construction of clothing space, we must take full account of the body's body characteristics, mainly due to the wearer's physical changes and movement will to some extent affect the clothing space, so clothing designers must pay full attention to this aspect. According to the characteristics of isomorphism, fashion designers need to be based on the body shape of the wearer changes, the effective design of its internal space. In the design of clothing space, the designer can fully use the pleated, the province and other technology, the maximum consistent with the body's body characteristics, and then fully show the wearer's body curve of the United States.

From the structural point of view, three-dimensional modeling of clothing must have the certain integrity. Through different combinations of forms, threedimensional modeling can be used to show the integrity of the geometry, while the full three-dimensional shape of the clothing can be a good combination of geometric decomposition of the figure out. Therefore, in the process of designing clothes, if designers want to improve the three-dimensional modeling of clothing, you can try a variety of graphics combinations, and organic combination of clothing space, to further improve and optimize the integrity of three-dimensional modeling of clothing. 


\section{3 clothing personality space analysis}

Clothing personality space refers to the internal space through the organic adjustment of clothing, the body of the wearer defects and shortcomings of a very good sound and make up, so that it can fully show the charm of the human body, the charm of clothing, and the two between the perfect combinations. Clothing personality space can be a good show of the uniqueness of human temperament and its own charm. If the wearer body is better, then clothing within the space between the fit and the human body is relatively high, showing the full beauty of the body; if the wearer body is poor, because the clothing has a certain space within the camouflage, it can be very good to cover up their physical defects and shortcomings. For example, in Western clothing, will make full use of clothing within the camouflage of space features, and effectively enhance the charm of the human body value and beauty.

If the clothing is static state, people see the general is the clothing of the plane form, and for the fashion designer, is to see the clothing before and after the three-dimensional and depth, with a certain dynamic elements. Through the action of the wearer, it can show the connotation of the inner space of the clothing, so that people can grasp and sense the connotation of the space inside the clothing in all directions.

In addition, the clothing for the corridor shape and internal space shape can reflect the same characteristics of the times. Through the wearer's clothing characteristics, people can be a good analysis and judge the wearer's time background, living conditions and ethnic and other aspects of the situation. Fashion designers through the display of its external shape and interior space characteristics, in the design process will be able to perfectly show the characteristics of the times.

The so-called clothing affinity refers to the space within the garment to provide the level of protection, and clothing outside the space and the perfect combination of space. Through the show of clothing affinity, can be well represented through the clothing of the natural atmosphere. People in the clothing design of health quality and natural desire, but also to a certain extent, fully demonstrated the pursuit of life. In order to better reflect the affinity of clothing, we must increase the effective interaction between people and clothing inside and outside the space, the maximum to meet the wearer in the physiological, psychological and sensory aspects of the demands. In addition, clothing affinity can also be through the clothing and the social environment between the full and harmonious show.

\section{Conclusion}

Overall, when the designer designs the costume, he should have correct understanding and deal with the relationship among clothing space, human and clothing design. In the design of three-dimensional modeling of clothing space, the most critical is the need to meet the wearer's comfort needs and aesthetic aspirations. Through the effective application of the three-dimensional space design elements, the space design art can be well displayed in the fashion design, 
and the wearer can understand and appreciate the artistic feeling more deeply and realize the pursuit of beauty.

\section{References}

[1] Tang Dongmei. Modeling Analysis of Three-dimensional Space Shape of Clothing, Art and Technology, 55(12), pp.68-70, 2011

[2] Zhang LongLin, Zhou Li, Anni. Three-dimensional Fabrication Technique of Fabrics in Dress Design. Textiles, 8 (5), pp.87- 91, 2013

[3] Wang Dan, Qiu PeiNa. Application of clothing space in three-dimensional modeling .Shandong Textile Economy, 12(10), pp. 18- 20, 2012

[4] Liu Jianqian, Chen Xiaoying. Modeling expression of garment graduation design based on interior space shape .Shandong Textile Economy, 9(6), pp.58- 61, 2011

[5] Zhao Ning. On the Three- dimensional Thinking in Fashion Design. Journal of Liaoning Economic Vocational Technological Institute, 8(4), pp.85-87, 2014 